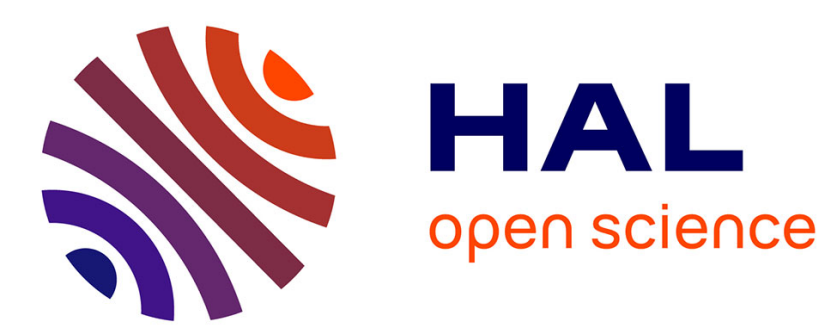

\title{
Few-Cycle Optical Solitons in Nonlinear Media: Spatiotemporal Collapse vs Bullet Cast
}

Igor V. Melnikov, Hervé Leblond, Dumitru Mihalache

\section{To cite this version:}

Igor V. Melnikov, Hervé Leblond, Dumitru Mihalache. Few-Cycle Optical Solitons in Nonlinear Media: Spatiotemporal Collapse vs Bullet Cast. Nonlinear Optics, Jul 2013, Kohala Coast, Hawaii, ÉtatsUnis. 10.1364/NLO.2013.NW4A.12 . hal-03208329

\section{HAL Id: hal-03208329 \\ https://univ-angers.hal.science/hal-03208329}

Submitted on 26 Apr 2021

HAL is a multi-disciplinary open access archive for the deposit and dissemination of scientific research documents, whether they are published or not. The documents may come from teaching and research institutions in France or abroad, or from public or private research centers.
L'archive ouverte pluridisciplinaire HAL, est destinée au dépôt et à la diffusion de documents scientifiques de niveau recherche, publiés ou non, émanant des établissements d'enseignement et de recherche français ou étrangers, des laboratoires publics ou privés. 


\title{
Few-Cycle Optical Solitons in Nonlinear Media: Spatiotemporal Collapse vs Bullet Cast
}

\author{
H. Leblond, ${ }^{(a)}$ I. V. Mel'nikov, ${ }^{(b)}$ and D. Mihalache ${ }^{(a, c, d)}$ \\ (a) Laboratoire de Photonique d'Angers, EA 4464, Université d'Angers, 2 Bd. Lavoisier, 49045 Angers Cedex 01, France \\ Departments of Electronic Materials and Microelectronics, National Research University of Electronic Technology, proezd 4806, \\ Zelenograd, Moscow 124498, Russian Federation \\ Horia Hulubei National Institute for Physics and Nuclear Engineering, 407 Atomistilor, Magurele-Bucharest 077125, Romania \\ (d) Academy of Romanian Scientists, 54 Splaiul Independentei, Bucharest 050094, Romania
}

\begin{abstract}
The spatiotemporal dynamics of few-cycle optical scalar and vector solitons in nonlinear Kerr media is studied outside the traditional framework of the SVEA. The pulse interactions, collapse threshold, and light bullets are calculated both analytically and numerically.
\end{abstract}

Keywords: Ultrafast nonlinear optics, Pulse propagation and solitons

In the recent years, there has been a great deal of interest in the area of ultraintense light pulses comprising merely a few optical cycles. These few-cycle pulses (FCPs) have both been widely exploited in ultrafast nonlinear optics and also pushed extensive modeling studies beyond the slowly-varying envelope approximation (SVEA). In particular, numerous applications speed up interest towards deep understanding the pulse self-compression down to the single-cycle pulsewidth that goes on in a transparent medium with instantaneous cubic (Kerr-like) nonlinearity. The dynamics of a FCP in a self-focusing medium can be described beyond the SVEA by means of using the modified Korteweg-de Vries equation (mKdV) [1], sineGordon (sG) [2,3] or mKdV-sG equations [4]. The $\mathrm{mKdV}$ and $\mathrm{sG}$ equations are completely integrable by the inverse scattering transform [5] whereas the $\mathrm{mKdV}-\mathrm{sG}$ equation is completely integrable only if conditions are imposed on the nonlinear properties of the medium [6]. All these equations admit breather solutions which can realistically describe the FCP solitons. In $(2+1)$ dimensions, the mKdV-based model should be replaced with the (non-integrable) generalized Kadomtsev-Petviashvili equation (GKPE) which, in turn, supplies a very distinct electrodynamical explanation for the stable few-cycle beam propagation $[7,8]$.

These findings have driven us to a specific question if the two-breather solution of the mKdV-sG equations describes the interaction in a Kerr-medium of two few-cycle optical solitons initially well separated, can be modeled in any optically reasonable setting. In this Report, we first consider the propagation of optical FCPs in a $(1+1) \mathrm{D}$ optical medium, such as a highly nonlinear optical waveguide. Our second goal is to expand this generic $\mathrm{mKdV}$-sG model onto a $(2+1) \mathrm{D}$ medium in such way to answer the practical question of optical collapse and possibility of its arrest for the FCP.

In any physical implementation, a train of FCPs can be launched into the medium in such a way that these propagate as a train of solitons. Due to the fluctuations of the intensity of the laser, the consecutive FCPs may have different energy/peak power, and, subsequently, different velocities. Therefore, it is only natural to expect them to overlap with each other and our goal is to predict what can happen during the interaction. Owing to the analytic solutions that describe the two-breather solution of the $\mathrm{mKdV}$-sG equation, we manage to define in explicit form, the amplitude, time location and shift resulting from the interaction.

In the $(2+1) \mathrm{D}$ case of a Kerr-like medium, the evolution equation (1) should be replaced with a cubic GKPE as (see [10])

$$
\left(U_{z}+\sigma_{1} U^{2} U_{\tau}+\sigma_{2} U_{\tau \tau \tau}\right)_{\tau}=U_{y y},
$$

where the dimensionless variables $U, z$, and $\tau$ are proportional to the electric field, propagation distance, and retarded time, correspondingly, and $y$ is proportional to the transverse coordinate. Notice that $U$ is not the amplitude of the FCP, but is proportional to the true electric field itself. The constants $\sigma_{l, 2}= \pm 1$ are related to the dispersion and nonlinearity properties of the medium; see, e.g., Refs. [2-4]. As an input FCP train we chose a linear superposition of the two-breather solution which we believe is the best fit for the two-cycle optical pulse. Since the final expressions are somewhat cumbersome, here we restrict ourselves to the highlight of the results obtained analytically.

Let us assume first that $\sigma_{1,2}=-1$, that is, the nonlinearity and dispersion yield temporal selfcompression while nonlinearity and diffraction tend to defocus the FCP and what is more memory effects come into action here. The peculiarities of such diffraction are displayed at Fig. 1 where the optical field is strongly localized in time domain yet considerably spatially delocalized. This typical crescent shape is due to the conjugated action of temporal self-compression and diffraction. If the joint effect of nonlinearity and diffraction is focusing $\left(\sigma_{1,2}=+1\right)$, and if the input is strong enough, the self-focusing might happen and two distinct regimes of the collapse are found numerically. At the same time, the spectrum of the FCP presents a strongly asymmetric oscillatory behavior that is in strict contrast with the case of the "long" pulses described within the SVEA. 
In other typical case (the so called short-wave limit), when the characteristic frequency of the optical transition is much smaller than the carrier frequency of the FCP, the spatiotemporal dynamics is described by a two dimensional sine-Gordon equation as [11]:

$$
U_{z \tau}=C \sin U+U_{y y},
$$

where $C$ depends on $z$ and is proportional to the inverse population.

a)

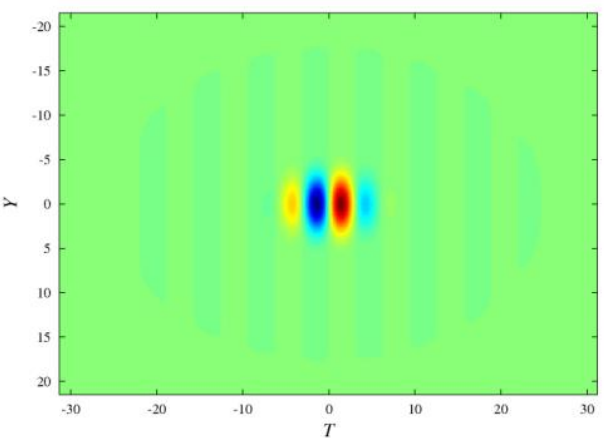

c)

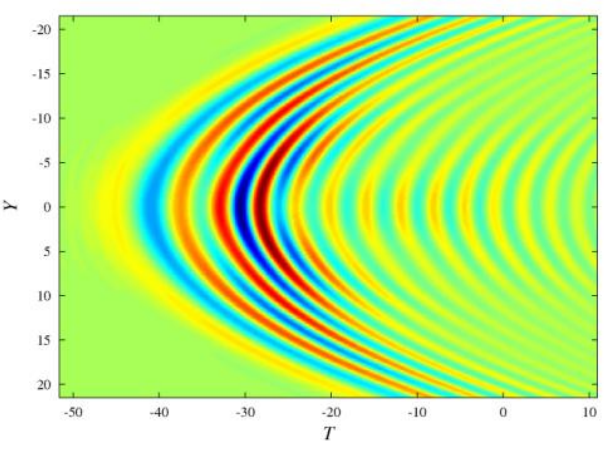

b)
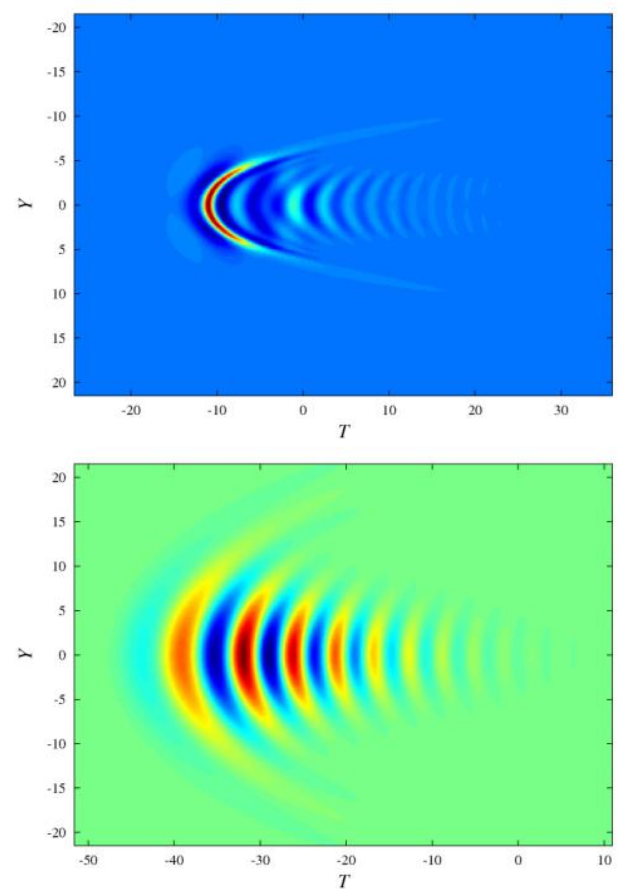

Figure 1 Nonlinear diffraction of the FCP: (a) input profile at the $(y, t)$-plane; (b) an intermediate stage with a typical crescent shape; (c) nonlinear diffraction; (d) linear diffraction.

The numerical simulations to Eq. (2) reveal evolution (not shown here) of the Gaussian pulse into a stable localized structure being affected neither by dispersion nor by diffraction; after a transient stage where the FCP radiates energy, its amplitude decreases and stabilization in the form of a localized oscillatory structure is reached eventually.

It is very much tempting to expand this type of a multiscale perturbative analysis to depart from the SVEA and to describe evolution of vector few-cycle optical solitons that propagate in, e.g., Kerr-like media. Hence, we take into account the vector character of the electric field and consider the spatiotemporal propagation effects properly. That is, in the long-range approximation we find that the vector field corresponding to such few-cycle solitons is adequately described by the two complex GKPEs (1) but coupled through the second term as $\sim\left(U^{2}+\right.$ $\left.V^{2}\right) U_{\tau \tau \tau}$, where $V$ is the field component orthogonal to $U$.

In conclusion, we study the spatiotemporal dynamics of few-cycle optical solitons by making use of the two-breather solution of the mKdV-sG equation (or GKPE-sG) found earlier [7, 9-11]. The shapes of input and output soliton envelopes as well as the phase and location shifts are computed by mean of the exact expressions for the four-soliton (two-breather) solutions of the $\mathrm{mKdV}-\mathrm{sG}$ equation. The remarkable feature is that, contrary to the case of the traditional (SVEA-type) soliton envelopes, no phase matching of any kind is required for the two-cycle pulses to interact efficiently. This interaction may bring novel features into the nonlinear propagation of trains of few-cycle optical pulses. At the same time, light bullet formation is proven for a medium, where the characteristic frequency of the optical transition is much smaller than the carrier frequency of the FCP. Contrary to that, the FCP propagation might also lead to the true wave collapse or nonlinear diffraction, depending on the sign of nonlinearity and dispersion. This Report completes our previous research efforts and presents a description for spatiotemporal dynamics of few-cycle scalar and vector optical solitons alternative to approaches based on the traditional slowly varying envelope (and phase) approximation. 


\section{References}

1. I. V. Mel'nikov et al., Phys. Rev. A 56, 1569 (1997).

2. H. Leblond and F. Sanchez, Phys. Rev. A 67, 013804 (2003).

3. I. V. Mel'nikov et al., IEEE J. Sel. Top. Quant. Electron. 10, 870 (2004).

4. H. Leblond et al., Phys. Rev. A 74, 063815 (2006).

5. R. K. Dodd et al., Solitons and Nonlinear Wave Equations (Academia, London, 1982)

6. K. Konno et al., J. Phys. Soc. Jpn. 37, 171 (1974)

7. I. V. Mel'nikov et al., Opt. Commun. 181, 345 (2000).

8. H. Leblond et al., Mathematics and Computers in Simulation 69, 378 (2005).

9. H. Leblond, I.V. Mel'nikov, and D. Mihalache, Phys. Rev. A 78, 043802 (2008).

10. H. Leblond, D. Kremer, and D. Mihalache, Phys. Rev. A 81, 033824 (2010).

11. H. Leblond and D. Mihalache, Phys. Rev. A 81, 063815 (2010). 